\title{
LC-MS/MS assay for the quantitation of the ribonucleotide reductase inhibitor triapine in human plasma
}

\author{
Julia Matsumoto a,b, Brian F. Kiesel ${ }^{\mathrm{a}, \mathrm{f}}$, Robert A. Parise ${ }^{\mathrm{a}}$, Jianxia Guo ${ }^{\mathrm{a}}$, Sarah Taylor ${ }^{\mathrm{c}}$, \\ Marilyn Huang c, Julie L. Eiseman ${ }^{\mathrm{a}, \mathrm{d}}$, S. Percy Ivy ${ }^{\mathrm{e}}$, Charles Kunos $^{\mathrm{e}}$, Edward Chu ${ }^{\mathrm{a}, \mathrm{d}}$, \\ Jan H. Beumer ${ }^{\mathrm{a}, \mathrm{d}, \mathrm{f}, *}$ \\ a Cancer Therapeutics Program, University of Pittsburgh Cancer Institute, Pittsburgh, PA, United States \\ ${ }^{\mathrm{b}}$ School of Pharmaceutical Sciences, Sao Paulo State University, Araraquara, SP, Brazil \\ ' Division of Gynecologic Oncology, Department of Obstetrics, Gynecology, and Reproductive Medicine, University of Pittsburgh-Magee Women's Hospital, \\ Pittsburgh, PA, United States \\ ${ }^{\mathrm{d}}$ Division of Hematology-Oncology, Department of Medicine, University of Pittsburgh School of Medicine, Pittsburgh, PA, United States \\ e Investigational Drug Branch, Cancer Therapy Evaluation Program, Division of Cancer Treatment and Diagnosis, National Cancer Institute, Bethesda, MD, \\ United States \\ ${ }^{\mathrm{f}}$ Department of Pharmaceutical Sciences, School of Pharmacy, University of Pittsburgh, Pittsburgh, PA, United States
}

\section{A R T I C L E I N F O}

\section{Article history:}

Received 29 June 2017

Received in revised form 18 August 2017

Accepted 28 August 2017

Available online 31 August 2017

\section{Keywords:}

Triapine

Tandem mass spectrometry

Assay

Validation

\begin{abstract}
A B S T R A C T
The ribonucleotide reductase inhibitor and radiosensitizer triapine (3-aminopyridine-2-carboxaldehyde thiosemicarbazone (3-AP), NSC 663249) is clinically being evaluated via the intravenous (IV) route for the treatment of cervical and vulvar cancer in combination with primary cisplatin chemoradiation. The need for a 2-h infusion and frequent administration of triapine is logistically challenging, prompting us to pursue oral (PO) administration. In support of the clinical trial investigating oral triapine in combination with chemoradiation, we developed and validated a novel LC-MS/MS assay for the quantification of triapine in $50 \mu \mathrm{L}$ human plasma. After protein precipitation, chromatographic separation of the supernatant was achieved with a Shodex ODP2 column and an isocratic acetonitrile-water mobile phase with $10 \%$ ammonium acetate. Detection with an ABI 4000 mass spectrometer utilized electrospray positive mode ionization. The assay was linear from 3 to $3,000 \mathrm{ng} / \mathrm{mL}$ and proved to be accurate $(97.1-103.1 \%)$ and precise $(<7.4 \% \mathrm{CV})$, and met the U.S. FDA guidance for bioanalytical method validation. This LC-MS/MS assay will be an essential tool to further define the pharmacokinetics and oral bioavailability of triapine.
\end{abstract} (c) 2017 Elsevier B.V. All rights reserved.

\section{Introduction}

In the United States, cervical cancer is the third most common gynecologic cancer with approximately 12,900 new cases and 4100 succumbing to disease per year [1]. Currently, chemoradiation with cisplatin weekly is used as first-line therapy for locally advanced disease in both cervical and vulvar cancers [2]. However, due to poor overall survival in patients with advanced stage disease, the use of novel agents and/or combinational therapies is needed to improve clinical outcomes.

\footnotetext{
* Corresponding author at: University of Pittsburgh Cancer Institute, Room G27E, Hillman Research Pavilion, 5117 Centre Avenue, Pittsburgh, PA 15213-1863, United States.

E-mail address: beumerj@gmail.com (J.H. Beumer).
}

Ribonucleotide reductase (RNR) enzyme inhibition has been known to sensitize both chemotherapy and radiation, which makes it an attractive target to combine with the current standard of care treatments. DNA synthesis and repair are dependent on the supply of deoxyribonucleoside triphosphates (dNTP) produced from RNR enzymes. RNRs maintain homeostasis by providing balanced production of dNTPs. The key role of RNRs in this process has been identified as a potential target of inhibition in a variety of human malignancies. Inhibitors have been developed that block the production of deoxynucleotides and are able to prevent cells from repairing and synthesising new DNA, triggering cellular senescence [3].

Gemcitabine and hydroxyurea are two cytotoxic agents that directly inhibit RNRs. Triapine is 1000 -fold more potent in inhibiting RNR than hydroxyurea, and it is a strong iron chelator that is able to deplete the cellular iron necessary for proper RNR function. 
RNR may not be the only target for triapine, with the $\mathrm{Fe}$ (Triapine) ${ }_{2}$ complex: (1) targeting mitochondrial function, depleting thioredoxin; (2) generating radical damage and reactive oxygen species; and (3) oxidizing cellular thiols [4]. In addition, iron has been found to play an important role in maintaining malignant tumor progression compared to normal cell function [5], which may explain, in part, the selective anticancer activity of triapine. The combination of triapine with radiation, which is currently being tested clinically, is based on observations of increased RNR expression after ionizing radiation and increased cell death upon combining triapine and ionizing radiation [6]. To date, over 30 clinical trials have been conducted to evaluate triapine alone or in combination with other cytotoxic agents and/or radiation therapy.

The combination of $3 \times$ weekly $25 \mathrm{mg} / \mathrm{m}^{2} 2$-h IV triapine with weekly $40 \mathrm{mg} / \mathrm{m}^{2}$ IV cisplatin and daily pelvic radiation in women with cervical cancer has been studied in phase I and II trials with clinical responses in 24 of 25 patients in the phase II trial [3,7]. The need for frequent administration of triapine and its 2-h infusion duration is logistically challenging, prompting us to pursue oral administration of triapine.

The initial assays for measuring triapine were based on methanol-EDTA based protein precipitation of serum, followed by ion-pairing reversed phase chromatography with UV detection at $400 \mathrm{~nm}$ at high flow rate $(1 \mathrm{~mL} / \mathrm{min})$ with a run time of $10 \mathrm{~min}$, an equilibration delay of $6 \mathrm{~min}$ with a dynamic range of $20-10,000 \mathrm{ng} / \mathrm{mL}$ [8-10]. The first reported LC-MS-MS assay [11] in plasma ranged from 1 to $1,000 \mathrm{ng} / \mathrm{mL}$ and utilized the chemically distinct 2-pyridinecarboxaldehyde as internal standard, separated on a reverse phase column by isocratic elution with $5 \%$ acetonitrile containing $0.2 \%$ acetic acid. Strong hydrogen bonding and chelating capabilities of triapine with proteins and metal ions in human plasma prompted the addition of EDTA ( $3 \mathrm{mM}$ ) to each sample to chelate metal ions prior to sample cleaning by cation exchange solid phase extraction. More recently, Feng et al. investigated triapine complexation behaviour and developed an LC-MS/MS assay utilizing the fluorine analogue 2-[(3-fluoro-2-pyridinyl)methylene] hydrazinecarbothioamide (NSC 266749) as internal standard [12]. Using this method, plasma samples were deproteinated with acetonitrile, dried down, and reconstituted. EDTA was used for dilution of stock solutions, and for reconstitution of dried down extracts to improve reproducibility and sensitivity of the detector response. and analytes were separated on a reversed phase column with a methanol-ammonium bicarbonate buffer mobile phase set at $\mathrm{pH} 8.5$, resulting in a 4 min run time and a linear calibration range of $0.250-50.0 \mathrm{ng} / \mathrm{mL}$. Plasma samples from mice administered triapine were analyzed to demonstrate the application of the assay.

In support of the clinical trial investigating oral triapine in combination with chemoradiation, we developed and validated an LC-MS/MS assay for the facile, sensitive quantification of triapine in human plasma. Our assay has a range of 3-3000 for an expected clinical concentration range of up to at least $500-1500 \mathrm{ng} / \mathrm{mL}$ as suggested by previous clinical data.

\section{Experimental}

\subsection{Chemicals and reagents}

Triapine $\left(M /\left(M+M_{+4}\right)>99.99 \%\right)$ was purchased from Selleckchem (Houston, TX). The internal standard, $\left[{ }^{13} \mathrm{C}_{3},{ }^{15} \mathrm{~N}\right]$-triapine $\left(M_{+4} /\left(M+M_{+4}\right)>99.99 \%\right)$, was purchased from Alsachim (Illkirch Graffenstaden, France). Acetonitrile, water (both HPLC grade), ammonium acetate, and dimethyl sulfoxide were purchased from Fisher Scientific (Fairlawn, NJ, USA). Control human plasma was purchased from Valley Biomedical (Winchester, VA, USA) and Lam- pire (Everett, PA, USA). PBS and Polyethylene glycol 400 (PEG400) was purchased from Fisher Scientific (Fairlawn, NJ, USA).

\subsection{Chromatography}

The LC system consisted of an Agilent (Palo Alto, CA, USA) 1200 SL autosampler and binary pump, a Shodex (New York, NY USA) ODP2 HP-2 B $(5 \mu \mathrm{m}, 50 \times 2 \mathrm{~mm})$ column, and an isocratic mobile phase. Mobile phase solvent $A$ consisted of acetonitrile $/ 10 \mathrm{mM}$ ammonium acetate $\mathrm{pH} 6.5(90 / 10, \mathrm{v} / \mathrm{v})$, and mobile phase solvent $\mathrm{B}$ consisted of water with $10 \mathrm{mM}$ ammonium acetate $\mathrm{pH}$ 6.5. The mobile phase composition was $85 \%$ solvent $A$ and $15 \%$ solvent $B$ pumped at $0.3 \mathrm{~mL} / \mathrm{min}$ from zero to $1.5 \mathrm{~min}$, followed by a rate of $0.6 \mathrm{~mL} / \mathrm{min}$ from 1.6 to $3 \mathrm{~min}$. The total run time was $3 \mathrm{~min}$.

\subsection{Mass spectrometry}

Mass spectrometric detection was carried out using ABI SCIEX (San Jose, CA, USA) 4000 hybrid linear ion trap tandem mass spectrometer with electrospray ionization in positive-ion, multiple reaction monitoring (MRM) mode. The settings of the mass spectrometer in positive mode scanning parameters were as follows: curtain gas 5 , IS voltage $50 \mathrm{~V}$, probe temperature $500^{\circ} \mathrm{C}$, GS1 40, GS2 40, declustering potential $30 \mathrm{~V}$, a collision energy of $30 \mathrm{~V}$, and an exit potential of $15 \mathrm{~V}$. Also, the temperature of the autosampler was $4{ }^{\circ} \mathrm{C}$. The MRM $\mathrm{m} / z$ transitions monitored were: $196.0>121.0$ for triapine and $200.0>125.0$ for $\left[{ }^{13} C_{3},{ }^{15} \mathrm{~N}\right]$-triapine. The LC system and mass spectrometer were controlled by Analyst software (version 1.4.2), and data were collected with the same software.

\subsection{Preparation of calibration standards and quality control samples}

Stock solutions of analyte triapine and internal standard $\left[{ }^{13} \mathrm{C}_{3},{ }^{15} \mathrm{~N}\right]$-triapine (see Fig. 1 for structures), were prepared independently at $1 \mathrm{mg} / \mathrm{mL}$ in dimethyl sulfoxide and stored at $-80^{\circ} \mathrm{C}$. A diluted $0.1 \mathrm{mg} / \mathrm{mL}$ stock solution was also prepared independently for both analyte and internal standard. These stock solutions were prepared in acetonitrile/water $(50 / 50, v / v)$. On the day of assay, the internal standard was diluted 1000 times and the analyte solution serially diluted (in steps of 10 -fold) in acetonitrile/water $(50 / 50, v / v)$ to obtain the lower calibration working solutions. These calibration working solutions were diluted in human plasma to produce the following analyte concentrations: 3, 10, 30, 100, 300, 1000, and $3000 \mathrm{ng} / \mathrm{mL}$. For each calibration series, zero and blank samples were also prepared from $50 \mu \mathrm{L}$ of control plasma.

Quality control (QC) stock solutions were stored at $-80^{\circ} \mathrm{C}$. These solutions were diluted in human plasma to produce the follow-
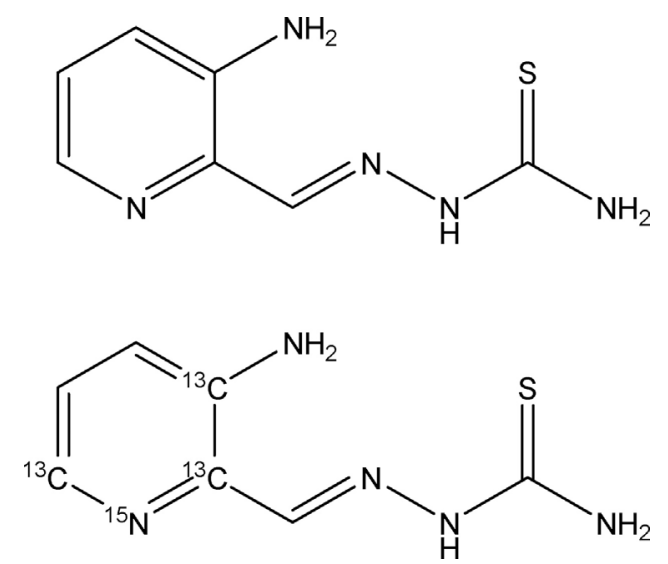

Fig. 1. Structures of triapine and $\left[{ }^{13} \mathrm{C}_{3},{ }^{15} \mathrm{~N}\right]$-triapine internal standard. 
ing QC samples of either: QC Lower Limit of Quantification (QCLL) $3 \mathrm{ng} / \mathrm{mL}$, QC Low (QCL) $5 \mathrm{ng} / \mathrm{mL}$; QC Mid (QCM) $100 \mathrm{ng} / \mathrm{mL}$, and QC High $(\mathrm{QCH}) 2500 \mathrm{ng} / \mathrm{mL}$.

\subsection{Sample preparation}

To each microcentrifuge tube were added $50 \mu \mathrm{L}$ of each sample (standard, QC or sample plasma), $10 \mu \mathrm{L}$ of $0.1 \mu \mathrm{g} / \mathrm{mL}$ $\left[{ }^{13} \mathrm{C}_{3},{ }^{15} \mathrm{~N}\right]$-triapine and $250 \mu \mathrm{L}$ of mobile phase A extraction mixture (acetonitrile with $10 \%$ ammonium acetate, pH 6.5). Samples were vortexed for $1 \mathrm{~min}$ on a Vortex Genie-2 set at 8 (Model G560 Scientific Industries, Bohemia, NY, USA) and then centrifuged at $13,000 \times g$ at room temperature for $10 \mathrm{~min}$. The resulting supernatants were transferred $(200 \mu \mathrm{L})$ to autosampler vials, followed by injection of $5 \mu \mathrm{L}$ into the LC-MS/MS system.

\subsection{Validation procedures}

\subsubsection{Calibration curve and lower limit of quantitation (LLQ)}

Decreasing concentrations of analytes were injected into the analytical system to determine the minimal concentration with a signal-to-noise ratio of at least 5:1. Calibration standards and blanks were prepared (see paragraph 2.4 and 2.5) and analyzed in triplicate to establish the calibration range with acceptable accuracy and precision. The analyte-to-internal standard ratio (response) was calculated for each sample by dividing the area of the analyte peak by the area of the internal standard peak. Standard curves were constructed individually by plotting the analyte-tointernal standard ratio versus the known concentrations in each sample. Standard curves were fitted by linear regression with weighting by $1 / y^{2}$, followed by back-calculation of concentrations. The deviations of these back-calculated concentrations from the nominal concentrations were expressed as percentage of the nominal concentration.

\subsubsection{Accuracy and precision}

The accuracy and precision of the assay were determined by analyzing samples at the $\mathrm{QCL}, \mathrm{QCM}$, and $\mathrm{QCH}$ concentrations in 6 replicates each in 3 analytical runs, together with independently prepared, triplicate calibration curves. Accuracy was calculated at each test concentration as: (mean measured concentration/nominal concentration) x $100 \%$.

Assay precision was calculated by ANOVA as previously described [13], by using SPSS 23.0 for Windows (SPSS Inc., Chicago, IL, USA). Back-calculated concentrations of calibration and QC samples were entered with the run number as factor. From the resulting mean squares of the within runs and mean squares of the between runs, the intra-assay and inter-assay precisions were calculated.

\subsubsection{Selectivity and specificity}

To investigate whether endogenous matrix constituents interfered with the assay, six individual batches of control, drug-free human plasma were processed and analyzed according to the described procedure. Responses of analytes at the LLQ concentrations were compared with the response of the blank samples. Cross-talk of triapine and $\left[{ }^{13} \mathrm{C}_{3},{ }^{15} \mathrm{~N}\right]$-triapine was characterized by detection in their respective MRM channels.

\subsubsection{Extraction recovery and matrix effect}

We determined the extraction recovery of triapine from plasma by comparing the absolute response of an extract of control plasma to which this analyte had been added after extraction, with the absolute response of an extract of plasma to which the same amount had been added before extraction. The matrix effect by plasma matrix components was defined as the decrease of the absolute response of an extract of control plasma to which analyte had been added after the extraction relative to the absolute response of reconstitution solvent to which the same amount of analyte had been added. Experiments were performed at the three QC concentrations, in quadruplicate.

\subsubsection{Stability}

Long-term stability experiments were performed in plasma and in stock solution after storage at $-80^{\circ} \mathrm{C}$. Stability in the stock solution was expressed as the percentage recovery of the stored solution ( 3 months) relative to a fresh solution. The stability of triapine in plasma at $-80^{\circ} \mathrm{C}$ was determined by assaying samples before and after storage. In addition, the stability of triapine in stock solution at room temperature for $6 \mathrm{~h}$ was determined in triplicate. All stability testing in plasma was performed in triplicate at the QCL, QCM and QCH concentrations. The effect of 3 freeze/thaw cycles analyte concentrations on plasma was evaluated by assaying samples after they had been frozen $\left(-80^{\circ} \mathrm{C}\right)$ and thawed on 3 separate days and comparing the results with those of freshly prepared samples. The stability of triapine in plasma during sample preparation was evaluated by assaying samples before and after $4 \mathrm{~h}$ of storage at room temperature. To evaluate the stability of triapine in reconstituted samples in the autosampler, we re-injected QC samples and calibration curves approximately $72 \mathrm{~h}$ after the first injection and compared the concentrations derived from the second injection with those derived from the first injection. The results of the second run were expressed as a percentage of their respective values in the first run.

\subsubsection{Dilutional integrity}

To demonstrate dilutional integrity, the ability to dilute samples from above the upper limit of quantitation to within the validated concentration range, plasma samples containing triapine above the upper limit of quantitation were diluted to within the assay range. Plasma samples $(\mathrm{N}=3)$ with analyte concentrations of $30,000 \mathrm{ng} / \mathrm{mL}$ were diluted 20 -fold (to $1500 \mathrm{ng} / \mathrm{mL}$ ) with control plasma and assayed.

\subsubsection{Cross validations}

To demonstrate the ability of our EDTA plasma-based assay to quantitate heparinized plasma samples, we quantitated our EDTA $\mathrm{QCL}, \mathrm{QCM}$, and $\mathrm{QCH}$ samples $(\mathrm{N}=4)$ against a heparinized plasma triplicate calibration curve.

To demonstrate the ability of our human plasma-based assay to quantitate murine plasma samples, we quantitated our human QCL, $\mathrm{QCM}$, and $\mathrm{QCH}$ samples $(\mathrm{N}=4)$ against a murine plasma triplicate calibration curve.

\subsection{Application of the assay}

As our clinical trial is on hold till manufacturing of drug supply has been completed, we demonstrated the application of the assay in mice. Samples obtained from mice intravenously dosed $20 \mathrm{mg} / \mathrm{kg}$ triapine were analyzed. Six female C57BL/6 mice were dosed with triapine and two mice were administered vehicle control (40\% PEG400/60\%PBS) at $10 \mu \mathrm{L} / \mathrm{g}$. Blood was collected at $5 \mathrm{~min}$ $(\mathrm{N}=3$ mice $)$ and $30 \mathrm{~min}(\mathrm{~N}=3$ mice $)$ time points using retro-orbital sinus bleeds with heparinized micro hematocrit capillary tubes followed by centrifugation $(12,000 \mathrm{xg}$ for $4 \mathrm{~min})$ to obtain plasma. Terminal samples in these same mice were collected at 60 and 120 min using cardiac puncture following euthanization with $\mathrm{CO}_{2}$ inhalation. Plasma was immediately stored at $-80^{\circ} \mathrm{C}$ upon collection until LC-MS/MS analysis. 
After triapine quantitation, non-compartmental analysis of pharmacokinetic parameters was performed using PK Solutions (Summit PK, Montrose, CO) on naively pooled data.

\section{Results and discussion}

\subsection{Development}

The method development of the assay included multiple variations in column type, extraction solvents, and HPLC gradients.

\subsubsection{Mass spectrometry}

The analyte was scanned in both negative and positive ionization modes until the most sensitive mode was identified for drug and internal standard. We determined that optimal sensitivity was achieved with positive ionization. The MRM transitions used have been reported previously $[11,12]$.

\subsubsection{Extraction and sample preparation}

The dilute-and-shoot method is simple to work with due to the reduced number of steps. However, it dilutes the amount of drug, which can adversely affect sensitivity. Therefore, different proportions and amounts of organic solvent were tested. Three extraction solvents were tested: acetonitrile, acetonitrile followed by ammonium acetate addition to the autosampler vial, and acetonitrile with $10 \%$ ammonium acetate $\mathrm{pH} 6.5$. The last solvent was chosen for protein precipitation due to the combination of best sensitivity and peak shape.

Triapine forms chelates with metal ions, including iron, and EDTA-plasma was chosen to reduce the potential loss of drug in the samples by such complexation. A plasma sample volume of $50 \mu \mathrm{L}$ was applied to keep solvent use low, resulting in a practical sample preparation executable in microtubes. In an additional experiment, the presence of $10 \mathrm{mM}$ ferric chloride decreased the mass spectrometric signal, and this effect was magnified by a drying down step, see Fig. 2. Although the presence of iron decreased the absolute peak area, the stable isotope internal standard tracked this behaviour, and the ratio of triapine to internal standard was not affected.

After appropriate chromatography was established with column 3 , see below, the possible plateauing of the response at higher concentrations due to detector saturation became limiting. This issue was resolved by varying the volume of plasma taken into preparation and volume injected into the mass spectrometer resulting in $500 \mu \mathrm{L}$ solvent added to $100 \mu \mathrm{L}$ of plasma, with injection of $10 \mu \mathrm{L}$. Upon switching to column 4 , see below, the assay proved to be more sensitive, and we subsequently modified the volumes to $250 \mu \mathrm{L}$ solvent added to $50 \mu \mathrm{L}$ of plasma and a $5 \mu \mathrm{L}$ injection volume, in effect halving the amount injected on column and reducing sample and solvent use. The internal standard amount added was modified to approximately $20 \mathrm{ng} / \mathrm{mL}$ triapine in plasma, which is the lower end of the calibration range.

\subsubsection{Chromatography}

We evaluated the following four columns: Synergi Polar RP $80 \mathrm{~A}(100 \times 2.0 \mathrm{~mm}, 4 \mu \mathrm{m})$, Synergi Hydro RP $80 \mathrm{~A}(100 \times 2.0 \mathrm{~mm}$, $4 \mu \mathrm{m})$, Asahi Pak NH2P-502D $(150 \times 2 \mathrm{~mm}, 5 \mu \mathrm{m})$ and ODP2 HP-2 B $(50 \times 2.0 \mathrm{~mm}, 5 \mu \mathrm{m})$. The first two columns did not allow adequate chromatographic behaviour due to bad peak shape, high background signal, or short retention time, even with a variation of mobile phases and gradients mobile phases tested: methanol/water, methanol/ammonium bicarbonate, acetonitrile/water, and acetonitrile/water with $0.1 \%$ formic acid.

The third column allowed chromatography based on the HILIC principle with water being the strong solvent [14], and this column was also tested with various mobile phases and gradients. A
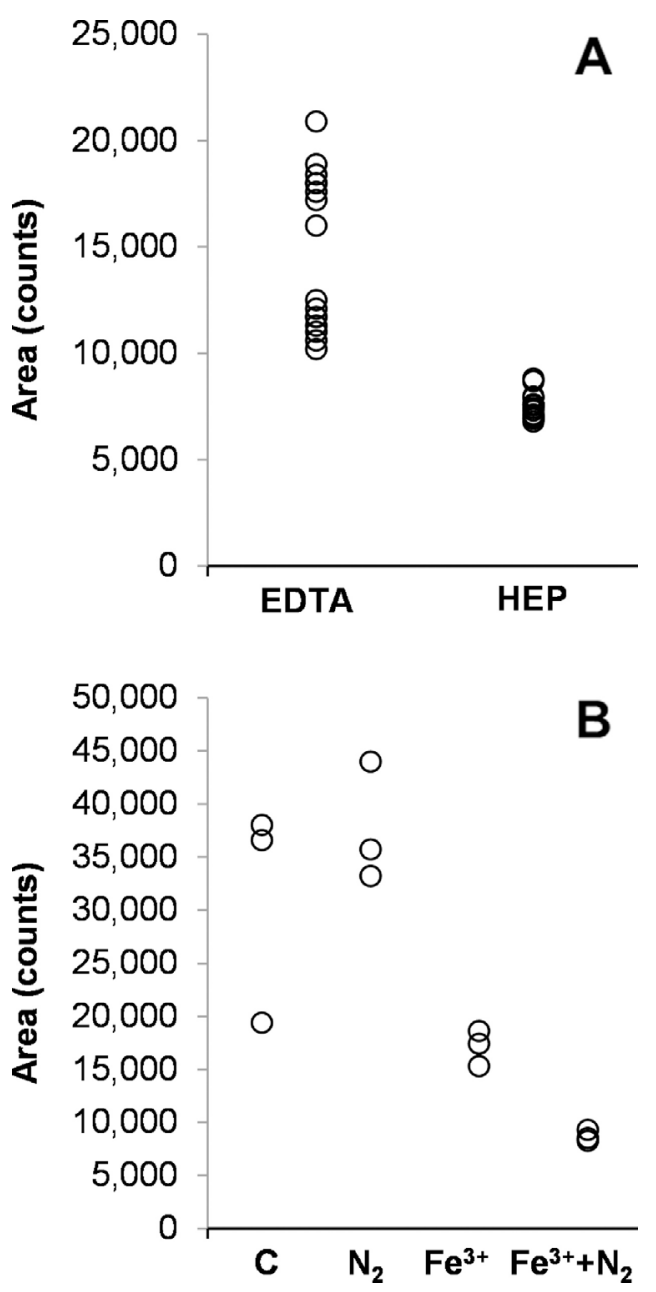

Fig. 2. (A) $\left[{ }^{13} \mathrm{C}_{3},{ }^{15} \mathrm{~N}\right]$-triapine internal standard peak area in calibration samples prepared in either EDTA or heparin anticoagulated plasma; (B) peak area of QCM samples processed as described (C), with a dry-down and reconstitution step $\left(\mathrm{N}_{2}\right)$, with the addition of $5 \mu \mathrm{L} 0.1 \mathrm{M} \mathrm{FeCl}_{3}\left(\mathrm{Fe}^{3+}\right)$, or with the addition of $5 \mu \mathrm{L} 0.1 \mathrm{M} \mathrm{FeCl}_{3}$ and a dry-down and reconstitution step $\left(\mathrm{Fe}^{3+}+\mathrm{N}_{2}\right)$.

mobile phase of acetonitrile/water with $0.1 \%$ formic acid (50/50 and $75 / 25, v / v)$ resulted in early elution $(1 \mathrm{~min})$. When the composition was changed to $90 / 10, v / v$, retention time increased $30 \mathrm{~s}$; however the triapine peak started to split. We next applied a gradient from $98 \%$ to $50 \%$ acetonitrile from 0 to $3 \mathrm{~min}$, held for another $2 \mathrm{~min}$, followed by re-equilibration, which resulted in 3 separate peaks; a small peak at $2.5 \mathrm{~min}$, a large peak at $5.6 \mathrm{~min}$, and a broadly eluting peak between 4 and $6 \mathrm{~min}$. A gradient elution in acetonitrile/water with $10 \mathrm{mM}$ ammonium acetate $\mathrm{pH} 5.5$ or $\mathrm{pH}$ 6.5 starting out with $95 \%$ acetonitrile resulted in a small and a large peak separated by approximately $1 \mathrm{~min}$. Isocratic elution at $70 \%$ acetonitrile followed by a wash phase resulted in a single symmetrical peak, but at the expense of retention. The peak shape, retention time ( $1.16 \mathrm{~min}$ ), and sensitivity (LLQ approximately $20 \mathrm{ng} / \mathrm{mL}$ ) were acceptable using this gradient, mobile phases and column.

In a final attempt to shorten the run time, we contacted the supplier of the NH2P column to enquire about shorter NH2P columns, and were prompted to evaluate the ODP2 column. This ultimately utilized column was perfused with acetonitrile with $10 \%$ ammonium acetate $\mathrm{pH} 6.5 /$ water with $10 \mathrm{mM}$ ammonium acetate as mobile phase components. In attempting to create more retention at lower acetonitrile content, the triapine signal again split in two peaks. After evaluating multiple gradients, we settled for a simple isocratic elution with a wash-step, as detailed above. The elution 
flow rate of $0.3 \mathrm{~mL} / \mathrm{min}$ with an increase to $0.6 \mathrm{~mL} / \mathrm{min}$ during the wash step allowed a 3 min run time. As detailed above, the new column resulted in a better sensitivity for triapine. Carry-over was assessed by injecting a prepared plasma sample with $30,000 \mathrm{ng} / \mathrm{mL}$ triapine, followed by serial plasma blank injections. Carry-over was less than $0.0025 \%$.

\subsection{Assay validation}

\subsubsection{Chromatography}

The approximate retention time of triapine was $0.7 \mathrm{~min}$ and of the internal standard, $\left[{ }^{13} \mathrm{C}_{3},{ }^{15} \mathrm{~N}\right]$-triapine, was also $0.7 \mathrm{~min}$. At a void volume of $0.5 \mathrm{~min}$, triapine had a capacity factor of 0.4 . Representative chromatograms of triapine (at the LLQ), and internal standards in plasma are displayed in Fig S1.

\subsubsection{Calibration curve and $L L Q$}

According to the FDA guidance for bioanalytical method validation [15], the calibration curve adequately describes the concentration versus response relationship if the observed deviation and precision are $\leq 20 \%$ for the LLQ and $\leq 15 \%$ for all other calibration concentrations. At least 4 of 6 calibration points should meet the above criteria [15].

The selected assay range of 3-3000 ng/mL fulfilled the FDA criteria for the LLQ concentration and the calibration curve. Accuracies and precisions at the different concentrations were determined from triplicate calibration curves on 5 separate days and are reported in Table S1. At most concentrations, the mean square of the within runs was greater than the mean square of the between runs, indicating that there was no significant additional variability due to the performance of the assay in different runs [13]. Representative calibration curves and corresponding correlation and regression coefficients are shown in Fig. S2.

\subsubsection{Accuracy and precision}

FDA guidance specifies that the accuracies for all tested concentrations should be within $\pm 15 \%$. Moreover, the precisions should not be $>15 \%$ CV except for the LLQ in which case these parameters should not exceed $20 \%$ [15].

The accuracies and intra- and inter-assay precisions for the tested concentrations ( $\mathrm{QCL}, \mathrm{QCM}$, and $\mathrm{QCH}$ ) were all within the defined acceptance criteria (Table S2).

\subsubsection{Selectivity and specificity}

According to FDA guidance, the signal at the LLQ must be at least 5 times the signal of any co-eluting peaks [15].

Chromatograms of six individual control plasma samples contained no co-eluting peaks $>20 \%$ of the analyte areas at the LLQ concentration (interference $<18.2 \%$ ) (Fig.S1).

Cross-talk calculations were performed and revealed that triapine cross-talks with the internal standard channel at approximately $0.0002 \%$ with identical retention times. The internal standard is added at approximately $20 \mathrm{ng} / \mathrm{mL}$, which would not be expected to result in meaningful interference with the triapine signal at the LLQ at $3 \mathrm{ng} / \mathrm{mL}$.

\subsubsection{Extraction recovery and matrix effect}

The FDA-guidance stipulates that extraction recovery be consistent and precise [15]. A recovery of $\geq 70 \%$ with a variation of $15 \%$ is generally accepted $[13,15]$. There is no specific requirement for matrix effect. Ultimately, the assay performance, as expressed in the precision and accuracy, is most relevant; however, a large and/or variable matrix effect may result in lack of assay robustness.

The recovery of triapine ranged from 90.7 to $110 \%$, with CVs between 9.7 and $12.1 \%$. Matrix effect ranged from 55 to $110 \%$ (i.e.

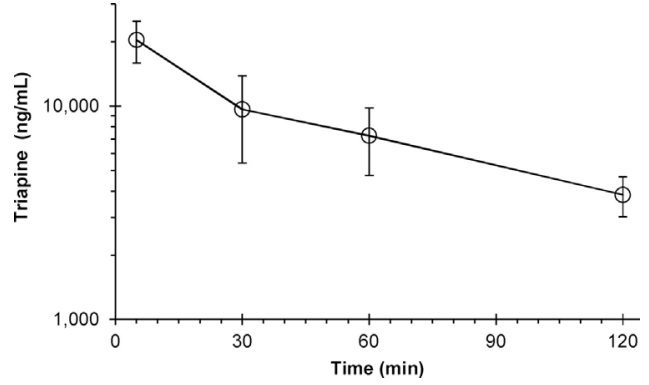

Fig. 3. Application of the assay. Concentration (mean $\pm S D, N=3$ ) vs time of triapine $(\bigcirc)$ in murine plasma $(\mathrm{ng} / \mathrm{mL})$ after $20 \mathrm{mg} / \mathrm{kg}$ IV administration of triapine.

ionization enhancement), with CVs between 5.0 and 11.1\% (Table S3).

\subsubsection{Stability}

Stability in biological samples is acceptable when $\geq 85 \%$ of the analyte is recovered. The stability of triapine stock solution at room temperature for $6 \mathrm{~h}$ was $99.4 \%$ (Table 1 ). Stability in stock solution for 4 months at $-80^{\circ} \mathrm{C}$ was $97.6 \%$. The stability of the analyte after 3 freeze thaw cycles $\left(-80^{\circ} \mathrm{C}\right.$ to RT) was between 99.7 and $103 \%$. Long-term stability ( 3 months) of the analyte in plasma at $-80^{\circ} \mathrm{C}$ was adequate with recovery between 94.1 and $99.8 \%$. The absolute responses of plasma extracts of triapine at the calibration concentrations, when reconstituted and kept in the autosampler for $72 \mathrm{~h}$, were $74.7-87.7 \%$ of the initial responses (CV 7.3-27.5\%), while the response of triapine relative to the internal standard signal ranged from 98.0 to $103.5 \%$ (CV 3.8-6.5\%). Importantly, the reinjection run passed the requirements of any run set by the FDA [7].

\subsubsection{Dilutional integrity}

The samples diluted from $30 \mu \mathrm{g} / \mathrm{mL}$ to $1500 \mathrm{ng} / \mathrm{mL}$ displayed $100.7 \%$ accuracy for triapine with a $\mathrm{CV}$ of $0.7 \%$. This result indicates dilutional integrity for triapine.

\subsubsection{Cross validations}

Accuracy and precision of back-calculated concentrations were within 15\% at QCL, QCM, and QCH concentrations. Results of EDTA vs heparin are provided in Table S4. Interestingly, although the back-calculated values and the response was not affected by the anticoagulant, the absolute peak areas in heparinized plasma were approximately half those in EDTA plasma (see Fig. 2), yet variability in absolute peak area varied less in heparinized plasma than in EDTA plasma.

Concentrations back-calculated using murine plasma resulted in accuracy and precision of within 15\% at QCL, QCM, and QCH concentrations (Table S5).

\subsection{Application of the assay}

Analyte concentrations quantitated in plasma after dosing mice with $20 \mathrm{mg} / \mathrm{kg}$ are depicted in Fig. 3. Appropriate dilutions placed all samples within the calibration range and no samples fell below the LLOQ.

Non-compartmental pharmacokinetic analysis of these samples revealed a half-life of $65 \mathrm{~min}$. Additional parameters included an $\mathrm{AUC}_{0-\mathrm{t}}$ of $1.08 \mathrm{mg} \mathrm{min} / \mathrm{mL} / \mathrm{kg}, \mathrm{AUC}_{0-\infty}$ of $1.44 \mathrm{mg} \mathrm{min} / \mathrm{mL} / \mathrm{kg}$, clearance of $13.8 \mathrm{~mL} / \mathrm{min} / \mathrm{kg}$, and volume of distribution at steady state of $1.15 \mathrm{~L} / \mathrm{kg}$. 
Table 1

Stability of triapine under varying conditions.

\begin{tabular}{|c|c|c|c|c|c|}
\hline Storage condition & \multicolumn{2}{|c|}{ Concentration (ng/mL) } & Stability (\%) & $\mathrm{CV}(\%)$ & Replicates \\
\hline \multicolumn{6}{|l|}{ Triapine } \\
\hline \multicolumn{6}{|l|}{ Stock solution $6 \mathrm{~h}$} \\
\hline Ambient temp. & & 100,000 & 99.4 & 4.4 & 3 \\
\hline \multicolumn{6}{|c|}{ Stock solution 4 months } \\
\hline$-80^{\circ} \mathrm{C}$ & & 100,000 & 97.6 & 4.5 & 3 \\
\hline \multicolumn{6}{|l|}{ Plasma $4 \mathrm{~h}$} \\
\hline \multirow[t]{3}{*}{ Ambient temp. } & QCL & 5 & 91.2 & 4.2 & 4 \\
\hline & QCM & 100 & 93.1 & 3.1 & 4 \\
\hline & $\mathrm{QCH}$ & 2500 & 96.5 & 5.8 & 4 \\
\hline \multicolumn{6}{|c|}{ Plasma 3 freeze-thaw cycles } \\
\hline \multirow[t]{3}{*}{$-80^{\circ} \mathrm{C}$} & QCL & 5 & 103.0 & 3.6 & 4 \\
\hline & QCM & 100 & 99.7 & 4.0 & 4 \\
\hline & $\mathrm{QCH}$ & 2500 & 100.9 & 5.4 & 4 \\
\hline \multicolumn{6}{|l|}{ Plasma 3 months } \\
\hline \multirow[t]{3}{*}{$-80^{\circ} \mathrm{C}$} & QCL & 5 & 94.1 & 11.4 & 4 \\
\hline & QCM & 100 & 99.8 & 6.0 & 4 \\
\hline & $\mathrm{QCH}$ & 2500 & 97.2 & 3.0 & 4 \\
\hline
\end{tabular}

\section{Conclusion}

The objective of the present study was to develop and validate a new analytical method for the quantitation of triapine in human plasma. Reversed-phase chromatography was used to identify triapine with triple quadrupole mass spectrometric detection. With triapine being a relatively polar compound, the short retention times on conventional reversed phase columns was predictable. Historically, we have had good experience with HILIC columns in achieving adequate retention for such compounds [14,16-18]. Unfortunately, triapine could not be eluted on our HILIC column without some degree of peak-splitting. Such effects are expected for sugars and other molecules that may exist in different steric conformations, and perhaps triapine, with or without complexation partners, also exists in some equilibrium of conformations which is kinetically too slow to behave as a single chromatographic entity on the HILIC column utilized $[4,19]$. Our assay is the first to utilize a stable isotope internal standard, which is expected to minimize variability due to a variety of factors including unforeseen complexation behaviour of triapine in individual clinical samples. Our development work also highlights the effects different anticoagulants can have on analyte signal. Although a stable isotope internal standard may appropriately correct for complexation and anticoagulant effects, maximizing signal likely increases robustness of the assay. When compared to the only other published LC-MS/MS assay (range $0.25-50 \mathrm{ng} / \mathrm{mL}$ [12]), our assay (range $3-3000 \mathrm{ng} / \mathrm{mL}$ ) avoids the need for frequent dilutions of clinical samples, which have been reported to reach $1500 \mathrm{ng} / \mathrm{mL}$. In addition, the previously reported assay [12] utilized EDTA throughout the various dilution steps to avoid triapine complexation effects, which is not required with our assay. The latter may be attributable to our use of a stable isotope internal standard, whereas the previous assay utilized a close analogue, which was chromatographically separated from triapine. Lastly, our assay is technically simpler without a drydown step and a slightly shorter run-time. Our assay allowed for the evaluation of triapine in mouse plasma, which is the first report of such data. Human reported PK parameter values are: clearance $43-58 \mathrm{~L} / \mathrm{h}[9,20]$, corresponding to $27-36 \mathrm{~L} / \mathrm{h} / \mathrm{m}^{2}$, compared to our murine clearance of $14 \mathrm{~mL} / \mathrm{min} / \mathrm{kg}$ corresponding to $2.5 \mathrm{~L} / \mathrm{h} / \mathrm{m}^{2}$; volume of distribution at steady state of $0.77 \mathrm{~L} / \mathrm{kg}$ [9] compared to our murine value of $1.15 \mathrm{~L} / \mathrm{kg}$; half-life of 55-63 min [9,20] compared to our murine value of $65 \mathrm{~min}$. Possibly, humans are capable of clearing triapine more extensively than mice, whereas the distribution volume is comparable.

In conclusion, we have developed and validated a rapid, facile assay for the quantitation of triapine in human plasma with a range of $3-3000 \mathrm{ng} / \mathrm{mL}$, adequately covering the expected clinical concentration range. By providing quantitative pharmacokinetic information, our assay will be a valuable tool to support further clinical development of triapine.

\section{Funding}

Support: Grant UM1-CA186690 (NCI-CTEP), R50 CA211241 (NCI). This project used the UPCI Cancer Pharmacokinetics and Pharmacodynamics Facility (CPPF) and was supported in part by award P30-CA47904. Additional support was provided by the Institute of International Education and CAPES/CNPq (JM).

\section{Appendix A. Supplementary data}

Supplementary data associated with this article can be found, in the online version, at http://dx.doi.org/10.1016/j.jpba.2017.08.036.

\section{References}

[1] R.L. Siegel, K.D. Miller, A. Jemal, Cancer statistics, 2015, CA: Cancer J. Clin. 65 (1) (2015) 5-29.

[2] N. Wang, Q.L. Guan, K. Wang, X. Zhou, C. Gao, H.T. Yang, T.G. Ni, Radiochemotherapy versus radiotherapy in locally advanced cervical cancer: a meta-analysis, Arch. Gynecol. Obstet. 283 (1) (2011) 103-108.

[3] C.A. Kunos, S. Waggoner, V. von Gruenigen, E. Eldermire, J. Pink, A. Dowlati, T.J. Kinsella, I trial of pelvic radiation, weekly cisplatin, and 3-aminopyridine-2-carboxaldehyde thiosemicarbazone (3-AP, NSC \#663249) for locally advanced cervical cancer, Clin. Cancer Res. 16 (4) (2010) 1298-1306.

[4] C.R. Chitambar, W.E. Antholine, Iron-targeting antitumor activity of gallium compounds and novel insights into triapine((R))-metal complexes, Antioxid. Redox Signal. 18 (8) (2013) 956-972.

[5] S.V. Torti, F.M. Torti, Ironing out cancer, Cancer Res. 71 (5) (2011) 1511-1514

[6] C.A. Kunos, S.M. Chiu, J. Pink, T.J. Kinsella, Modulating radiation resistance by inhibiting ribonucleotide reductase in cancers with virally or mutationally silenced p53 protein, Radiat. Res. 172 (6) (2009) 666-676.

[7] C.A. Kunos, T. Radivoyevitch, S. Waggoner, R. Debernardo, K. Zanotti, K. Resnick, N. Fusco, R. Adams, R. Redline, P. Faulhaber, A. Dowlati, Radiochemotherapy plus 3-aminopyridine-2-carboxaldehyde thiosemicarbazone (3-AP, NSC \#663249) in advanced-stage cervical and vaginal cancers, Gynecol. Oncol. 130 (1) (2013) 75-80.

[8] L. Feun, M. Modiano, K. Lee, J. Mao, A. Marini, N. Savaraj, P. Plezia, B. Almassian, E. Colacino, J. Fischer, S. MacDonald, Phase I and pharmacokinetic study of 3-aminopyridine-2-carboxaldehyde thiosemicarbazone (3-AP) using a single intravenous dose schedule, Cancer Chemother. Pharmacol. 50 (3) (2002) 223-229.

[9] J. Murren, M. Modiano, C. Clairmont, P. Lambert, N. Savaraj, T. Doyle, M. Sznol, Phase I and pharmacokinetic study of triapine, a potent ribonucleotide reductase inhibitor, administered daily for five days in patients with advanced solid tumors, Clini. Cancer Res. 9 (11) (2003) 4092-4100.

[10] F.J. Giles, P.M. Fracasso, H.M. Kantarjian, J.E. Cortes, R.A. Brown, S. Verstovsek, Y. Alvarado, D.A. Thomas, S. Faderl, G. Garcia-Manero, L.P. Wright, T. Samson, A. Cahill, P. Lambert, W. Plunkett, M. Sznol, J.F. DiPersio, V. Gandhi, Phase I and pharmacodynamic study of Triapine, a novel ribonucleotide reductase 
inhibitor, in patients with advanced leukemia, Leuk. Res. 27 (12) (2003) 1077-1083

[11] A. Mortazavi, Y. Ling, L.K. Martin, L. Wei, M.A. Phelps, Z. Liu, E.J. Harper, S.P. Ivy, X. Wu, B.S. Zhou, X. Liu, D. Deam, J.P. Monk, W.J. Hicks, Y. Yen, G.A. Otterson, M.R. Grever, T. Bekaii-Saab, A phase I study of prolonged infusion of triapine in combination with fixed dose rate gemcitabine in patients with advanced solid tumors, Invest. New Drugs 31 (3) (2013) 685-695.

[12] Y. Feng, C.A. Kunos, Y. Xu, Determination of triapine, a ribonucleotide reductase inhibitor, in human plasma by liquid chromatography tandem mass spectrometry, Biomed. Chromatogr. BMC (2015).

[13] H. Rosing, W.Y. Man, E. Doyle, A. Bult, J.H. Beijnen, Bioanalytical liquid chromatographic method validation. a review of current practices and procedures, J. Liq. Chromatogr. Relat.Technol. 23 (3) (2000) 329-354.

[14] J.H. Beumer, E. Joseph, M.J. Egorin, J.M. Covey, J.L. Eiseman, Quantitative determination of zebularine (NSC 309132), a DNA methyltransferase inhibitor, and three metabolites in murine plasma by high-performance liquid chromatography coupled with on-line radioactivity detection, J. Chromatogr. B: Analyt. Technol. Biomed. Life Sci. 831 (1-2) (2006) 147-155.

[15] U.S. Department of Health and Human Services Food and Drug Administration, Guidance for Industry-Bioanalytical Method Validation, U.S.Department of Health and Human Services, Food and Drug Administration; Center for Drug Evaluation; and Research Center for Veterinary Medicine, 2001.
[16] R.A. Parise, M.J. Egorin, J.L. Eiseman, E. Joseph, J.M. Covey, J.H. Beumer, Quantitative determination of the cytidine deaminase inhibitor tetrahydrouridine (THU) in mouse plasma by liquid chromatography/electrospray ionization tandem mass spectrometry, Rapid Commun. Mass Spectrom. ARCM 21 (13) (2007) 1991-1997.

[17] J.H. Beumer, J.L. Eiseman, R.A. Parise, E. Joseph, J.M. Covey, M.J. Egorin, Modulation of gemcitabine (2',2'-difluoro-2'-deoxycytidine) pharmacokinetics, metabolism, and bioavailability in mice by 3,4,5,6-tetrahydrouridine, Clin. Cancer Res. 14 (11) (2008) 3529-3535.

[18] J.L. Holleran, J.L. Eiseman, R.A. Parise, S. Kummar, J.H. Beumer, LC-MS/MS assay for the quantitation of FdCyd and its metabolites FdUrd and FU in human plasma, J. Pharm. Biomed. Anal. 129 (2016) 359-366.

[19] D. Kotoni, I. D’Acquarica, A. Ciogli, C. Villani, D. Capitani, F. Gasparrini, Design and evaluation of hydrolytically stable bidentate urea-type stationary phases for hydrophilic interaction chromatography, J. Chromatogr. A 1232 (2012) $196-211$.

[20] C.A. Kunos, E. Chu, J.H. Beumer, M. Sznol, S.P. Ivy, Phase I trial of daily triapine in combination with cisplatin chemotherapy for advanced-stage malignancies, Cancer Chemother. Pharmacol. 79 (1) (2017) 201-207. 